\title{
Cervical Esophagotomy for Removal of an Ingested Clam Shell: A Very Uncommon Foreign Body Ingestion
}

\author{
Edoardo Virgilio Diletta Giuliani Alice Nigro Marcello Gasparrini \\ Genoveffa Balducci
}

Medical and Surgical Sciences and Translational Medicine, Faculty of Medicine and Psychology "Sapienza,"

St. Andrea Hospital, Rome, Italy

\section{Significance of the Study}

- The clam shell was retrieved by performing cervical esophagotomy and the patient recovered well. Clinicians should be aware of this particular type of foreign body ingestion because it necessitates prompt surgery rather than nonoperative management or endoscopy.

\section{Keywords}

Foreign body ingestion · Clam shell · Esophagotomy

\section{Abstract}

Objective: To report the removal of an ingested clam shell that was firmly impacted in the esophagus. Clinical Presentation and Intervention: A 77-year-old man presented at our hospital with acute dysphagia after eating a seafood risotto. An urgent dedicated examination (noncontrast helical multislice computed tomography scan of the neck and flexible esophagoscopy) detected a clam shell lodged in the upper esophagus. After several unsuccessful endoscopic attempts, a lifesaving cervical esophagotomy was performed and the foreign body was retrieved. Conclusion: This patient who ingested clam shell recovered well following the retrieval of the foreign body by performing a lifesaving cervical esophagotomy.

(c) 2017 S. Karger AG, Basel

\section{KARGER}

E-Mail karger@karger.com www.karger.com/mpp
(C) 2017 S. Karger AG, Basel

Karger Open access

This is an Open Access article licensed under the terms of the Creative Commons Attribution-NonCommercial 3.0 Unported license (CC BY-NC) (www.karger.com/OA-license), applicable to the online version of the article only. Distribution permitted for non-commercial purposes only.

\section{Introduction}

The ingestion of a foreign body with impaction in the esophagus is a common gastrointestinal emergency $[1,2]$. A myriad of usual as well as infrequent objects have been reported $[1,2]$. Clam shell ingestion represents a very unusual occurrence; as of 2017, only 6 cases have been described in the literature. Differently from other materials, the retrieval of this bivalve mollusk can entail very serious medical and surgical problems. We report the successful removal of a clam shell by cervical esophagotomy.

\section{Case Report}

A 77-year-old man presented with acute dysphagia after eating a seafood risotto. His previous medical history was significant for cervical spine fixation performed 24 years previously because of amyotrophic lateral sclerosis. At the visit, apart from the oropharyngeal discomfort, the patient was eupneic and hemodynamically stable. A noncontrast helical multislice computed tomography

Edoardo Virgilio, MD, PhD

St. Andrea Hospital

via di Grottarossa 1035-39

IT-00189 Rome (Italy)

E-Mail aresedo1992@yahoo.it 

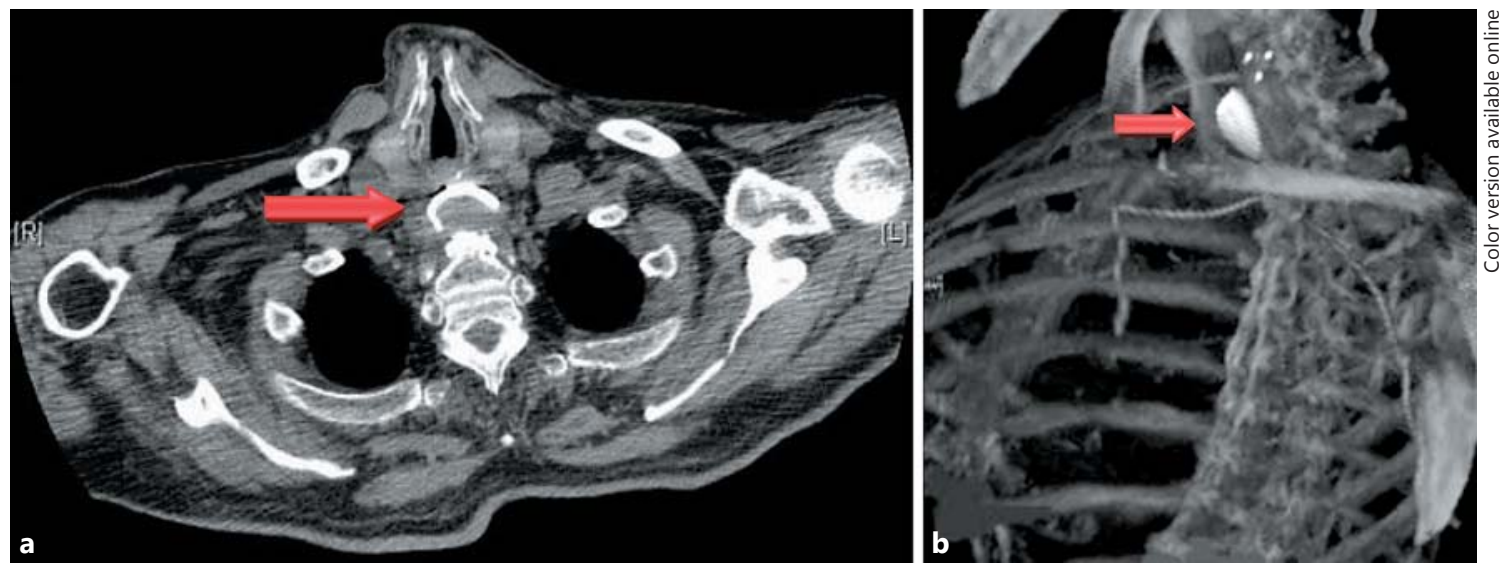

Fig. 1. a Noncontrast cervical computed tomography (CT) scan showed a hyperdense object with a concave morphology and a diameter of $4 \mathrm{~cm}$ lying in the upper esophagus at the subglottis level anteriorly to C6-C8 (red arrow). b The CT sagittal reconstruction outlined a maritime design congruent with a clam shell (red arrow).

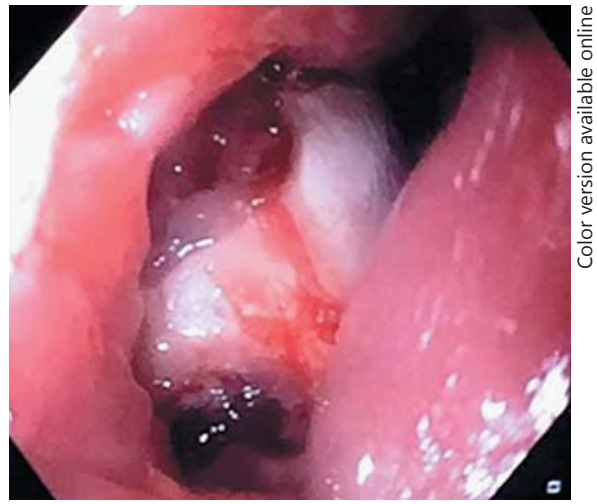

Fig. 2. At esophagoscopy, a whitish clam shell impacted in the cervical esophagus was confirmed as the foreign body.

(CT) scan of the neck was performed urgently: a radio-opaque foreign body with a concave morphology and a diameter of $4 \mathrm{~cm}$ was seen lodged in the upper esophagus at the subglottis level anteriorly to C6-C8 (Fig. 1a). Imaging sagittal reconstruction outlined a maritime design congruent with the shell of a clam (Fig. 1b). An immediate flexible endoscopy was performed that confirmed a whitish clam shell as the foreign body (Fig. 2); however, it failed to retrieve the object due to its particularly milled surface. Three flexible esophagoscopies were attempted subsequently but were all unsuccessful. Thereafter, a rigid endoscopy was carried out but the removal maneuvers also failed. At day 25 after ingestion, the patient developed acute respiratory failure that required lifesaving orotracheal intubation. At this point, surgery was designated as the only feasible treatment to retrieve the object through a left lateral cervicotomy. A longitudinal esophagotomy was accomplished along the Laimer-Killian triangle, permitting the extraction of the clam shell once split in 2 pieces (Fig. 3). The postoperative course was unremarkable, and the patient was discharged home after a few days and advised to maintain a regular oral diet.

Cervical Esophagotomy for Removal of an Ingested Clam Shell

\section{Discussion}

In general, foreign body ingestion with impaction in the esophagus is a common gastrointestinal emergency resulting from accidental or intentional action [1]. On the other hand, accidental ingestion of a clam shell is an unusual event. As of 2017, in fact, after burrowing into the world literature, we have found only 2 other cases dealing with clam shell ingested and impacted in the esophagus $[3,4]$. In one case a $4.5-\mathrm{cm}$ clam valve was removed via rigid esophagoscopy (after an unsuccessful flexible endoscopy), and in the other the successful retrieval (at the third endoscopy) was complicated by esophageal perforation and tension pneumothorax requiring emergency chest tube and repair of the perforation $[3,4]$. Differently from the aforementioned instances, our patient had medical history significant for a serious neurodegenerative disease (amyotrophic lateral sclerosis) which required an operation of cervical spine fixation 24 years previously. In addition to the considerable diameter of the bivalve $(4 \mathrm{~cm})$, which could probably entail problems even for healthy people, we do think that both of these components (long-standing Lou Gehrig disease and former neurosurgical intervention) could have participated in this adverse clinical phenomenon. The former, in fact, could have caused discoordinated swallowing leading to the bivalve ingestion, whereas the latter could have created some fibrotic sequelae involving the cervical esophagus (extraluminal anatomic distortion and/or mucosal stricture) contributing to the endoluminal impaction. For our patient, 4 consecutive flexible esophagoscopies and 1 rigid upper endoscopy were attempted in a very short period

Med Princ Pract 2017;26:390-392 DOI: $10.1159 / 000477403$ 


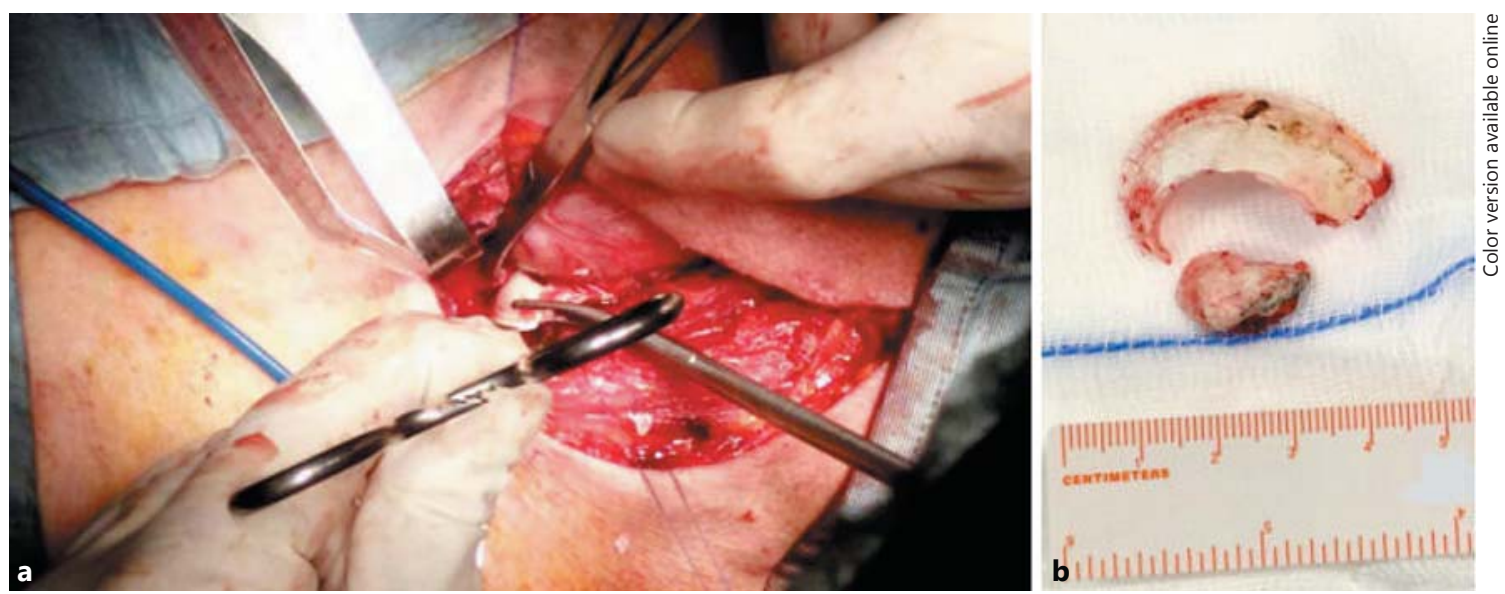

Fig. 3. a Through a left lateral cervicotomy, a longitudinal esophagotomy was accomplished along the LaimerKillian triangle permitting the extraction of the clam shell split in 2 pieces. $\mathbf{b}$ The periostracum of the shell measured $4 \mathrm{~cm}$ in diameter.

of time; however, all practices were unsuccessful. Hence, considering the patient's poor health condition (acute respiratory insufficiency requiring orotracheal intubation), surgery appeared to be the only possible curative option. And so it was. Indeed, cervical esophagotomy accomplished at day 25 permitted retrieval of the shell and, secondarily, amelioration of the general healthy status. Our treatment strategy was conducted in keeping with the American Society of Gastrointestinal Endoscopy Guidelines which indicate surgery for those initially asymptomatic cases of immobile blunt foreign bodies complicated by clinical deterioration, perforation, and sepsis $[2,5]$.

There are also other circumstances describing a clam shell passing in the small bowel and causing intestinal perforation or subocclusion. In the first event open sur- gery is mandatory, while in the latter extracorporeal shock wave lithotripsy has been successfully described twice [6-8]. Furthermore, evidence exists that the ingestion of a clam shell should dictate immediate retrieval rather than simple clinical or radiological observation due to the ascertained risk of developing the food proteininduced enterocolitis syndrome [9].

\section{Conclusion}

This was a case of successful retrieval of an ingested clan shell by lifesaving cervical esophagotomy. Such ingestion requires prompt surgery rather than nonoperative management or endoscopy.

\section{References}

1 Triadafilopoulos G, Roorda A, Akiyama J: Update on foreign bodies in the esophagus: diagnosis and management. Curr Gastroenterol Rep 2013;15:317.

2 Tseng HJ, Hanna TK, Shuaib W, et al: Imaging foreign bodies: ingested, aspirated, and inserted. Ann Emerg Med 2015;66:570-582.

3 Pelucchi S, Bianchini C, Ciorba A, et al: Unusual foreign body in the upper cervical oesophagus: case report. Acta Otorhinolaryngol Ital 2007;27:38-40.
4 Mato Ponce M, Muñoz Alcántara M, Pérez Pérez A, et al: Tension pneumothorax during removal of a foreign body from the esophagus (in Spanish). Rev Esp Anestesiol Reanim 2001;48:151-154.

5 Syrakos T, Zacharakis E, Antonitsis P, et al: Surgical intervention for gastrointestinal foreign bodies in adults: a case series. Med Princ Pract 2008; 17:276-279.

6 Tomas X, Alos L, Cores E, et al: Small bowel perforation due to ingested clam valve: imaging findings. Eur Radiol 2005;15:189-190.
7 Ramón Dalmau M, Ibarz Servio L, Mauri Cunill A, et al: Extracorporeal shockwave lithotripsy on a clam shell fragment lodged in the ileocecal valve. Arch Esp Urol 1994;47:159-161.

8 Vicens C, Nadal A, Ponseti JM, et al: Application of extracorporeal lithotripsy in intestinal subocclusion caused by a clam shell (in Spanish). Rev Esp Enferm Dig 1994;86:612-614.

9 Hayashi D, Aoki T, Shibata R, et al: Case of food protein-induced enterocolitis syndrome caused by short-neck clam ingestion (in Japanese). Arerugi 2010;59:1628-1633. 